\title{
Norois
}

Environnement, aménagement, société

204 | 2007/3

Patrimoine, culture et construction identitaire dans les territoires ruraux

\section{Touchart (Laurent) (dir.), Géographie de l'étang. Des théories globales aux pratiques locales}

Paris, L'Harmattan, 2007, 228 p.

Denis Mercier

(2) OpenEdition

Journals

Édition électronique

URL : http://journals.openedition.org/norois/1846

DOI : $10.4000 /$ norois. 1846

ISBN : 978-2-7535-1552-9

ISSN : 1760-8546

Éditeur

Presses universitaires de Rennes

Édition imprimée

Date de publication : 1 septembre 2007

Pagination : 95-97

ISBN : 978-2-7535-0533-9

ISSN : 0029-182X

Référence électronique

Denis Mercier, «Touchart (Laurent) (dir.), Géographie de l'étang. Des théories globales aux pratiques locales », Norois [En ligne], 204 | 2007/3, mis en ligne le 01 septembre 2007, consulté le 22 septembre 2020. URL : http://journals.openedition.org/norois/1846 ; DOI : https://doi.org/10.4000/norois. 1846

(c) Tous droits réservés 


\title{
COMPTE RENDU BIBLIOGRAPHIQUE
}

\author{
Touchart (Laurent) (dir.), 2007, Géographie de l'étang. Des théories globales aux \\ pratiques locales, Paris, L'Harmattan, 228 p.
}

Professeur à l'université d'Orléans et éminent spécialiste français des lacs et zones humides, Laurent Touchart a réuni les contributions de sept de ses collègues géographes, biologistes, gestionnaires, dans un ouvrage dense de 228 pages sur les étangs. Car l'étang, objet géographique répond à de nombreux enjeux. Il doit à la fois assouvir une demande de valorisation économique et faire l'objet d'aménagements afin d'assurer ses fonctions d'épuration des eaux fluviales, d'écrêtage de crue, de soutien d'étiage. Mais, l'étang est aussi enjeux patrimoniaux, paysage à conserver, support d'activités diverses et tisse du lien social.

L'ouvrage est construit en trois parties et huit chapitres. La première, intitulée « L'étang et les définitions à travers le monde : de la France marécageuse à la glose en marge du texte », regroupe trois chapitres. Le premier écrit par Laurent Touchart s’intéresse à

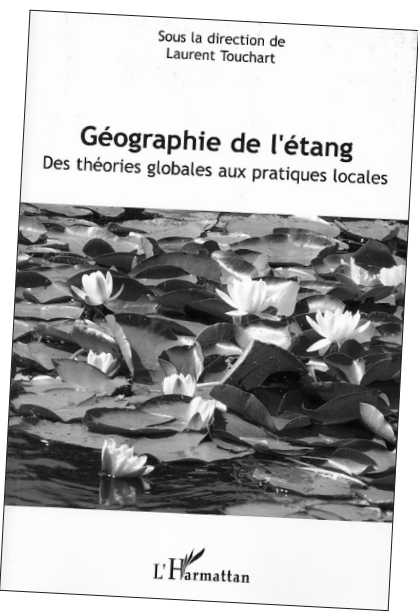
la définition de l'étang en géographie limnologique.

Les étangs font partie de la grande famille des zones humides dont la vocation est avant tout piscicole. Ce sont des plans d'eau continentaux artificiels, " dont la taille intermédiaire entre la mare et le lac permet une structuration irrégulière et temporaire des masses d'eau à l'échelle de quelques jours, une inertie se développant face à son encadrement immédiat (limnosystème de versant) ». Ou dit autrement « un plan d'eau intérieur de taille moyenne, dont les masses d'eau s'individualisent à l'échelle de quelques jours ». Laurent Touchart nous livre un premier chapitre dense à la recherche d'une définition géographique, en jouant avec habilité sur la variation des échelles spatiales et temporelles. Il nous offre à nouveau une synthèse magistrale à partir de ses lectures polyglottes. Le lecteur retiendra facilement la différence entre l'étang, le lac et la mare, grâce au sens pédagogique très développé de l'auteur, car, qu'on se le dise : "l'étang fait la grimace », " le lac fait la tête » et "la mare fait les petits yeux » (rendez-vous p. 43, figure 2).

Dans un autre style, Claude Genest nous rappelle (chapitre 2) que le mot étang dérive du latin stagnum, qui stagne. Le terme est ensuite transformé en ancien français en estanc, estanche : étendue d'eau dont un barrage empêche l'écoulement. Ses recherches dictionnairiques, mais surtout l'apport des praticiens québécois, livrent des informations précieuses sur les multiples usages des étangs : approvisionnement en eau potable, aquiculture (élevage en eau douce), régulation des inondations, irrigation, lieu d'habitat pour la faune (oiseaux aquatiques...), purification des eaux usées en milieu urbain, industriel ou rural, plan d'eau récréatif. Il nous donne une définition simple de l'étang: « retenue d'eau douce construite par l'homme, en tout ou en partie, qui sert à combler différents besoins (agriculture, génie, tourisme, éducation) » et une définition développée, que le lecteur retrouvera page 80.

Ensuite, dans le troisième chapitre, Pascal Bartout et Céline Bernard s’interrogent sur la définition de l'étang comme outil de gestion. Les deux auteurs nous apportent 
des précisions par rapport aux deux premiers chapitres. Un étang est « un objet artificiel car muni d'un système de vidange », sa fonction première est «l'approvisionnement en poissons ». Sa morphologie est précisée : «profondeur entre 0,7 et 3 mètres et une superficie comprise entre 1 et plus de 60 hectares ». Pour Damien Banas (chap. 4), la superficie moyenne des étangs français est de 3,7 ha. L'analyse des usages des étangs par « les sociétés stagnustres » de l'époque moderne, révèle une pratique multifonctionnelle optimisée, mais sans gestion organisée. Alors que présentement, les étangs font l'objet d'une «volonté gestionnelle, mais en l'absence de compréhension et d'individualisation des conséquences potentielles des différents usages des étangs ». Ce qui amène les auteurs à la conclusion suivante : «À vouloir rentabiliser à tout prix son étang, que ce soit financièrement ou psychologiquement, celui-ci ne combine plus les usages multiples qui en faisaient son essence même. Ceci entraîne des dysfonctionnements graves. »

Les chapitres rédigés ensuite par Damien Banas sur la sédimentation des étangs, Laurent Touchart sur la température de l'eau et ses enjeux et Françoise Ardillier-Carras sur l'environnement humain de l'étang, composent la deuxième partie intitulée "l'étang et son milieu : des effets à maîtriser, des obstacles à surmonter».

Les étangs fonctionnant comme des pièges à sédiments, ou " boîtes noires ", Damien Banas étudie les répercussions écologiques, notamment le rôle des MES et de la turbidité, sur la productivité primaire et la biomasse phytoplanctonique. L'origine des sédiments et leurs transports primaires et secondaires sont également évoqués de même que les méthodes d'analyse (carottage, datation, profil bathymétrique, trappes...).

La connaissance de la température de l'eau des étangs apparaît comme « un enjeu considérable ", selon Laurent Touchart, qui y consacre un chapitre 5 copieux, dense et magnifiquement illustré par des cartes de zonation thermique et des profils thermiques de premières mains, notamment à partir de l'étang des Oussines qu'il étudia de 1997 à 2005 sur la haute Vézère en Limousin. L’approche naturaliste est privilégiée pour l'acquisition des données thermiques et leur traitement géomatique permet la réalisation de cartes. La température de l'eau est considérée ici comme un indice de l'état sanitaire d'un étang et donc des rivières qui en dépendent. Des chiffres précis sur le réchauffement des eaux des étangs sont donnés à différentes échelles temporelles et spatiales. Ils apportent ainsi des éléments novateurs pour les gestionnaires des bassins.

Les étangs répondant à des finalités économiques, ils deviennent ainsi au cœur de relations sociales faites de convivialité et parfois de conflits d'usage. C'est ce qu'étudie par enquêtes directes, Françoise Ardillier-Carras dans le chapitre 6, avec comme support spatial les étangs du Limousin. Les résultats montrent que ces derniers ont aujourd'hui essentiellement une fonction récréative, tout en gardant une finalité agricole, alors qu'ils servaient initialement à subvenir aux besoins alimentaires.

La troisième partie, intitulée «Les étangs et les échelles géographiques : régions d'étangs, étangs de lieux » se compose de deux chapitres.

Le chapitre 7 «Armagnac et Limousin, deux régions oubliées de l'histoire des étangs », rédigé par Alexandra Angéliaume-Descamps et Bascal Bartout, veut réhabiliter l'histoire de ces étendues d'eau par une étude des archives depuis le XVIII siècle. Dans les deux régions, « la pisciculture ne constitue pas la principale finalité des étangs, n'en a jamais formé l'élément exclusif au cours de l'histoire ». La meunerie, puis maintenant l'irrigation et l'agrément constituent leur valorisation.

Dans le dernier chapitre «Un étang dans la ville : la réserve naturelle périurbaine de Saint-Quentin-en-Yvelines » rédigé par Alain Morand, c'est de l'étang de Trappes, une des pièces maîtresses du système hydraulique des eaux de Versailles, dont il est question. Cet étang de 120 hectares a cinq fonctions : régulation des crues, assainissement, loisirs nautiques, pêche, préservation de la biodiversité. Comment cohabitent 
toutes ces attributions? «En relative bonne intelligence » selon les conclusions de cette étude. Un exemple de gestion à transposer sur bien d'autres territoires sensibles de l'hexagone...

Si l'ouvrage est introduit par son coordonnateur, Laurent Touchart, il manque, comme très souvent dans les ouvrages collectifs, une conclusion. Sur le plan formel, un index thématique, mais hélas non géographique, vient clore l'ouvrage. L'ensemble est bien écrit. Certaines illustrations photographiques, trop réduites, occupent moins de place que leurs légendes! Les éditions L'harmattan avaient octroyé à Laurent Touchart en 2000 plus d'espace aux photographies dans son ouvrage sur l'origine et la morphologie des lacs. C'est regrettable. Cet ouvrage de grande qualité est à lire et surtout à faire connaître, bien au-delà de la sphère des géographes, car il démontre tout l'intérêt des études géographiques pour la gestion de nos territoires.

Denis MERCIER 
